\title{
Comprendre les symptômes éprouvés par les sujets atteints du cancer du poumon
}

\section{Par Catherine A. Kiteley et Margaret I. Fitch \\ Résumé}

Le but de cette étude était d'acquérir une meilleure compréhension des sentiments des patients envers leurs symptômes du cancer du poumon, lors de la première consultation dans un centre régional de cancérologie, et deux mois plus tard. Seize patients ont été interrogés à deux occasions sur les symptômes qu'ils éprouvaient et sur ce qui leur causait la plus grande gêne. On leur a également demandé de décrire les différentes stratégies qu'ils adoptaient pour gérer ces symptômes. Les symptômes les plus couramment mentionnés étaient la fatigue et la douleur. Les participants ont déclaré devoir faire face simultanément à plusieurs symptômes; ils ont expliqué comment ces symptômes s'intensifiaient sur la durée et ont indiqué recourir à très peu de stratégies de gestion des symptômes. Ils ont qualifié ces symptômes de gênants car ils interfèrent avec les activités de la vie quotidienne ou entraînent des réactions émotionnelles ou cognitives. La fatigue était souvent jugée la plus gênante. Ces travaux apportent de nouveaux éléments de réflexion sur la manière dont sont conduits les évaluations des patients et l'écoute dont bénéficient ces derniers de la part des professionnels de santé. La description que donnent les patients de leurs symptômes et des stratégies qu'ils utilisent suggère qu'ils sont souvent indissociables du vécu quotidien du cancer du poumon.

Le cancer du poumon est la première cause de mortalité liée au cancer au Canada et représente $30 \%$ des décès par cancer chez les hommes et $25 \%$ chez les femmes (INCC, 2005). Malgré les avancées thérapeutiques, le taux de survie à 5 ans pour un cancer pulmonaire non à petites cellules est de $10 \%$ à $15 \%$ et de $2 \%$ à $5 \%$ pour un cancer pulmonaire à petites cellules (Richardson et Johnson, 1993).

Chez la majorité des patients atteints d'un cancer du poumon, la trajectoire de la maladie est rapide et fatale, caractérisée par le développement et la progression accélérée des symptômes. À mesure que les symptômes apparaissent et s'aggravent, les patients rencontrent des difficultés à accomplir leurs activités quotidiennes. Les infirmières sont les mieux placées pour aider les patients et leur famille à gérer les symptômes associés à l'évolution de la maladie. Cependant, afin que les infirmières puissent déterminer le type d'assistance le mieux adapté aux patients atteints de cancer du poumon, une information sur le vécu de ces derniers est nécessaire. À l'heure actuelle, les données décrivant la perception que les patients ont des symptômes qui accompagnent leur maladie sont insuffisantes. Nous ne savons que peu de chose sur les symptômes que les patients eux-mêmes considèrent les plus gênants. La majorité de la littérature existante décrit les symptômes du point de vue des soignants.

Plusieurs chercheurs ont suggéré que les infirmières pouvaient avoir une perception des symptômes associés au cancer différente de celle des patients (Grossman, Sheidler, Swedeen, Mucenski et Piantadosi, 1991; Holmes et Eburn, 1989). Cette connaissance incomplète de la perception du patient risque d'empêcher les soignants d'aborder les problèmes qui préoccupent ce dernier et de baser leurs interventions sur leurs propres perceptions, sans aucune validation de la part du patient. Cerner la perspective du patient sur les symptômes du cancer du poumon est une intervention nécessaire qui permet aux infirmières de disposer d'une information complète dans ce domaine.

\section{Objectifs}

Le but de l'étude est de décrire les perceptions des symptômes telles que rapportées par les patients atteints d'un cancer du poumon et ce, tout au long de leur maladie. Nous voulons mieux comprendre quels sont les symptômes éprouvés par les patients, quels sont ceux qui leur causent le plus d'inconfort, et les stratégies de soins qu'ils utilisent pour les gérer. Préalablement à cette étude longitudinale, nous avons élaboré une étude pilote pour évaluer le recrutement des participants et le guide d'entrevue. C'est sur cette étude pilote que porte le présent article qui s'intéresse à l'entrevue initiale effectuée au moment du diagnostic et à la seconde entrevue, deux mois plus tard. Les enseignements issus de ces travaux contribueront à la préparation de l'étude longitudinale avec un échantillon plus large.

\section{Publications de référence}

La perspective du patient sur le vécu des symptômes du cancer du poumon n'a pas fait l'objet de recherches approfondies. Il existe divers rapports sur l'incidence et la prévalence des symptômes du cancer du poumon tout au long de la maladie, mais leurs conclusions varient selon les chercheurs (Brown, Carrieri, JansonBjerklie et Dodd, 1986; Burt, O’Driscoll, Notley, Barber et Stout, 1990; Hollen, Gralla, Kris, Eberly et Cox, 1999). Les patients atteints d'un cancer du poumon éprouvent un certain nombre de symptômes directement liés à leur maladie, tels la dyspnée, la toux, des douleurs thoraciques, une respiration sifflante (DeMaria et Cohen, 1987; Edmonds, Karlsen, Khan et Addington-Hall, 2001) et l'hémoptysie (Coy et Kennelly, 1980; Hollen et al., 1999). La fatigue, la perte d'appétit, la perte de poids et les troubles du sommeil font également partie des symptômes dits plus généraux (Blesch et al., 1991; Lutz, Huang, Ferguson, Kavanagh, Tercilla et Lu, 1997). Malheureusement, les comparaisons en matière d'incidence et de prévalence entre ces études sont difficiles étant donné les différentes approches des chercheurs et les variations des caractéristiques des échantillons concernés (Marino, Zoppi, Morelli, Buoncristiano et Pagni, 1986; Degner et Sloan, 1995; Bailey, Parma et Stephens, 2000).

Quelques rares chercheurs se sont penchés sur la détresse ressentie par les patients atteints d'un cancer du poumon à l'égard de leurs symptômes. McCorkle et Quint-Benoliel (1983) ont étudié la détresse rapportée par les patients $(n=56)$ peu après le diagnostic initial. La fatigue était signalée comme étant le symptôme le plus pénible, suivie par la modification de l'appétit et la douleur. Sarna (1993) rapporte que la fatigue, la douleur et l'insomnie étaient perçues par les patients comme les malaises les plus pénibles, alors que Tishelman, Degner et Mueller (2000) placent la fatigue, la confiance dans l'avenir (incertitude), et l'insomnie parmi les plus pénibles. Pour Lobchuk,

Catherine Kiteley, inf., MScInf., travaille au Credit Valley Hospital de Mississauga, Ontario.

Margaret I. Fitch, inf., PhD, est directrice de l'Unité de recherche psychosociale et comportementale du Centre régional de cancérologie de Toronto-Sunnybrook, Toronto, Ontario. Courriel : marg.fitch@sw.ca 
Krisjanson, Degner, Blood et Sloan (1997), la fatigue, la toux et la douleur atteignent les scores les plus élevés de la détresse liée aux symptômes.

Afin de concevoir des interventions adaptées aux symptômes vécus par les patients atteints d'un cancer du poumon, les infirmières ont besoin de mieux comprendre comment ces symptômes sont perçus par les patients. L'enquête réalisée auprès de malades ambulatoires, récemment inscrits auprès d'un centre régional de cancérologie, a pour but de répondre aux questions suivantes : 1) quels sont les symptômes que vous éprouvez? 2) quelles sont les formes les plus courantes de ces symptômes et quel est l'impact de ces derniers? 3) qu'est-ce qui contribue à atténuer ou à exacerber les symptômes? et 4) quels symptômes percevez-vous comme étant les plus gênants?

\section{Méthodologie}

Cette étude pilote descriptive utilise des entrevues semi-structurées, laissant aux participants la liberté de décrire leurs expériences personnelles avec n'importe quel symptôme, avant de se voir soumettre une liste de symptômes prédéterminés. La liste prédéterminée garantit à tous les patients la possibilité de répondre au même stimulus.

Après avoir obtenu l'approbation déontologique du Service de la recherche de l'Université de Toronto et du Comité d'éthique de la recherche du Sunnybrook and Women's Health Sciences Centre, les participants ont été recrutés dans le service de pneumologie d'un centre régional de cancérologie, selon les critères d'admissibilité suivants : 1) patient nouvellement enregistré,2) diagnostic confirmé de cancer du poumon, 3) pas de déficience cognitive ni d'antécédents psychiatriques majeurs, 4) maîtrise suffisante de la langue anglaise, 5) pleine conscience du diagnostic, et 6) résidant dans la région métropolitaine de Toronto. Les personnes ayant accepté de participer ont été interrogées à deux reprises, dans les deux semaines de leur première visite dans le centre de cancérologie (Temps 1) et deux mois plus tard (Temps 2). Toutes les entrevues ont été conduites et enregistrées sur bande audio par la même investigatrice (CK) et se sont tenues dans les lieux choisis par les participants.

\section{Guide d'entrevue}

Le guide d'entrevue était divisé en deux parties. La première partie consistait en une question ouverte suivie de quatre questions de clarification. L'entrevue débutait par une question non structurée (p. ex. « Comment vous êtes-vous senti(e) ces derniers jours? ») afin de donner aux participants la possibilité d'exprimer ce qui leur tenait le plus à coeur. Le participant était convié à décrire chaque symptôme qui lui venait spontanément à l'esprit, à expliquer ce qui le déclenchait, comment il le gérait et quelles étaient les stratégies de soins efficaces. La deuxième partie du guide d'entrevue consistait en des questions fermées, se référant à la littérature consacrée aux symptômes du cancer du poumon et concernant l'hémoptysie, l'essoufflement, la perte d'appétit, la fatigue, la respiration sifflante, la perte de poids, la douleur, la toux, l'insomnie et le mucus. La liste fournissait des réponses normalisées, tout en laissant aux participants la possibilité d'ajouter des symptômes qu'ils n'avaient pas encore décrits. Pour chaque nouveau symptôme, les quatre questions de clarification étaient posées.

Les questions finales de l'entrevue portaient sur l'identification du symptôme que les patients jugeaient le plus incommodant et leur demandaient des renseignements démographiques et médicaux ainsi que d'autres commentaires. Les renseignements démographiques concernaient l'âge, le sexe, la situation matrimoniale, le statut professionnel, les conditions de logement, les revenus du ménage et le niveau d'éducation. Les données sur la maladie et le traitement incluaient les affections concomitantes, la prise d'oxygène, les antécédents tabagiques et le temps écoulé depuis le diagnostic. Le dossier médical du patient a fourni des précisions additionnelles sur le stade de la maladie, le traitement suivi, la médication actuelle et la date du dernier traitement. Avant la seconde entrevue, des renseignements ont été recueillis sur le traitement suivi depuis l'entrevue précédente (c.-à-d. le type de traitement, les hospitalisations, les consultations d'un médecin, les consultations d'une infirmière et la médication actuelle).

\section{Analyse}

L'analyse a consisté en une analyse statistique descriptive et de contenu (Polit et Hungler, 2003; Weber, 1990). Les entrevues ont été transcrites mot pour mot et lues plusieurs fois, de manière à bien saisir les réponses des participants. Pour répondre aux questions de recherche 1, 3 et 4, chaque dossier a été examiné ligne par ligne afin de dégager les informations importantes et d'établir une liste des symptômes mentionnés, des facteurs aggravants pour chacun d'entre eux, des interventions de gestion des symptômes par le patient, des actions jugées efficaces, ainsi que du symptôme jugé le plus incommodant. La fréquence de chaque symptôme a été mesurée pour l'ensemble des participants. L'analyse de contenu a permis de dégager les types de symptômes et leur impact (question 2). Chaque compte rendu textuel a été étudié une nouvelle fois afin de bien peser le sens des mots et des phrases et d'en dégager la signification. Les items de même contenu ont été regroupés et placés dans une catégorie qui leur correspondait.

Les comptes rendus des deux entrevues avec quatre participants ont été confiés à la deuxième investigatrice, qui a appliqué la même méthode d'analyse pour les questions 1, 3 et 4. Pour la question 2 en deux parties, elle avait à sa disposition les étiquettes des catégories avec les items placés sous chacune d'elles. La deuxième investigatrice a dressé les mêmes listes de symptômes pour les quatre participants à l'instar de la première investigatrice et confirmé que toutes les données de chaque entrevue avaient été utilisées. Elle a approuvé la catégorisation de $90 \%$ des items de l'analyse de contenu. Les deux investigatrices ont passé en revue les items sujets à controverse et discuté ensemble jusqu'à ce qu'elles atteignent un consensus sur la catégorie qui leur convenait. La première investigatrice s'est ensuite chargée de l'analyse des entrevues des 12 sujets restants.

\section{Résultats}

On a vérifié l'admissibilité à l'étude de soixante-quinze patients. Trente-cinq ne répondaient pas aux critères d'éligibilité, à cause du lieu de résidence $(\mathrm{n}=18)$, de la langue $(\mathrm{n}=9)$, de confusion mentale $(n=4)$, d'antécédents psychiatriques $(n=2)$, et d'un diagnostic non confirmé $(\mathrm{n}=2)$. Sur les 42 personnes admissibles, 22 ont décliné l'invitation parce qu'elles ne voyaient pas l'intérêt de participer $(n=12)$, étaient bouleversées par le diagnostic $(n=6)$, éprouvaient une détresse liée aux symptômes $(n=3)$ ou à cause d'un refus de la famille $(\mathrm{n}=1)$. Sur les 20 participants entendus initialement, 16 ont complété l'entretien deux mois plus tard. Quatre ne se sont pas présentés à la deuxième entrevue, pour cause de décès $(n=2)$ ou parce qu'ils se sentaient « trop mal » $(n=2)$. Cet article porte sur les 16 sujets qui ont pris part aux deux entrevues. La durée des entrevues variait de 30 à 90 minutes et était de 60 minutes pour la majorité.

\section{Caractéristiques démographiques choisies}

Neuf hommes et sept femmes ont participé à l'étude. Treize étaient mariés et la plupart $(n=15)$ vivaient avec leur conjoint ou d'autres membres de la famille. L'âge moyen des participants était de 66,5 ans (étendue de 50 à 89 , écart-type $=10,06$ ). Onze ont déclaré disposer d'un revenu familial annuel brut inférieur à 40000 \$. Tous les participants ont signalé des antécédents tabagiques récents, et 7 étaient toujours fumeurs. L'un des participants était sous oxygène au moment de la première entrevue. En plus du cancer du poumon, 8 participants ont déclaré d'autres troubles médicaux (p. ex. problèmes cardiaques, problèmes gastro-intestinaux, antécédents de diabète, cécité, sciatique).

Lors de la première entrevue, les participants ont déclaré prendre une moyenne de 4,3 médicaments par jour (étendue 1-16, écarttype $=3,6$ ), et 5 (étendue $1-16$, écart-type $=3,4$ ) au moment de la 
seconde entrevue. Les analgésiques étaient le médicament plus fréquemment cité. Quatre participants avaient suivi une chimiothérapie avant la première entrevue, alors que la majorité $(n=14)$ avait reçu un traitement entre les deux entrevues, (9 avaient subi une radiothérapie de la poitrine, 2 avaient eu une radiothérapie et une chimiothérapie, 1 avait subi une chirurgie thoracique et une radiothérapie, 1 une chirurgie thoracique seule, et 1 une chimiothérapie uniquement).

\section{Les symptômes éprouvés}

À l'occasion des deux entrevues, les participants ont dégagé certains symptômes spontanément pendant la première partie et en ont révélé certains autres durant la seconde partie de l'entrevue, à l'incitation de l'investigatrice. Ils ont ainsi mentionné 21 symptômes différents dans le cadre de la première entrevue (moyenne $=6,3$; écarttype $=2,6$; étendue 2-11) et 29 durant la seconde (moyenne=7,3; écarttype $=3,11$; étendue 3-13). Les symptômes rapportés par plus de 25 $\%$ des participants incluaient l'essoufflement, la perte d'appétit, la fatigue, la respiration sifflante, la perte de poids, la douleur, la toux, l'insomnie et le mucus (voir le tableau 1). Une large gamme de symptômes n'était rapportée que par un ou deux participants (c.-à-d. indigestion, modification du goût, sensation de tête légère, sentiment d'anxiété, faiblesse des jambes, diminution de la production de salive, nycturie, fièvre, pieds enflés, mal de gorge, brûlures dues aux radiations, baisse de la libido, équilibre chancelant, chute de cheveux, doigts engourdis, sentiment de déprime, etc.). La fatigue était le symptôme le plus souvent cité au cours des deux entrevues. Le nombre de symptômes rapporté par la majorité des participants différait entre les deux entrevues. Lors de la seconde entrevue, huit des participants ont énuméré davantage de symptômes tandis que cinq autres ont dit éprouver moins de symptômes. Tous, à l'exception de deux participants, ont décrit au moins un symptôme différent de ceux qu'ils avaient mentionnés la première fois. Dans l'ensemble, environ deux tiers des symptômes ont été dégagés spontanément, le reste à la demande de l'investigatrice.

\section{Facteurs précipitants}

Lors des deux entrevues, beaucoup de participants n'ont fourni que des réponses brèves sur les facteurs précipitants. La plupart ont mentionné un seul facteur précipitant pour chaque symptôme ou ont expliqué que rien ne venait aggraver celui-ci. « Faire quelque chose » était le facteur qualifié le plus souvent cité par les patients. Les participants ont expliqué comment les activités d'intérieur et d'extérieur (c.-à-d. jardiner, pelleter la neige, faire les tâches ménagères, monter les escaliers ou faire de la marche) provoquaient la douleur, la fatigue et l'essoufflement. Ils ont souvent attribué l'aggravation d'un symptôme à la présence d'un autre. Par exemple, le mucus rendait la toux plus pénible pour quatre participants, alors que l'anxiété causait chez d'autres des problèmes de sommeil. Les participants étaient généralement incapables de dégager ce qui faisait augmenter la perte d'appétit, la respiration sifflante, le mucus et la perte de poids.

\section{Types les plus communs et impact des symptômes}

Les participants ont proposé diverses descriptions de types et de l'impact des symptômes. Ces descriptions peuvent se résumer à quatre catégories établies durant l'analyse : combinaisons de symptômes, caractéristiques des symptômes, impact des symptômes sur la vie de tous les jours, et symptômes déclenchant une réponse cognitive/émotionnelle.

Combinaisons de symptômes. Lorsque les participants parlaient de leurs symptômes, ils disaient en ressentir certains séparément, tandis que d'autres se manifestaient ensemble. Tous les participants ont évoqué au moins un symptôme qui se manifestait seul (la fatigue, l'essoufflement, la perte d'appétit, la perte de poids, la douleur, l'insomnie, la respiration sifflante, le mucus), mais la majorité lors de la première entrevue $(n=10)$ et de la seconde $(n=9)$ a mentionné des symptômes en relation avec d'autres symptômes. Les combinaisons décrites le plus souvent dans la première entrevue comprenaient les symptômes respiratoires (c.-à-d. respiration sifflante, toux, mucus et essoufflement, $n=6)$; et la fatigue associée à l'essoufflement $(n=4)$. Au cours de la seconde entrevue, les combinaisons de symptômes respiratoires $(n=5)$ et de la perte de poids avec la perte d'appétit $(n=5)$ étaient plus souvent évoquées.

Les symptômes étaient souvent décrits comme ayant une action réciproque, et la discussion autour d'un symptôme amenait souvent les patients à parler d'un autre symptôme. Nombre de participants ne faisaient pas de distinction ni de séparation nettes entre un symptôme et un autre. Selon les mots de l'un d'entre eux

La respiration sifflante... ça fait s'essouffler... Tout est étroitement lié d'une manière ou d'une autre... pour expectorer le mucus quand vous en avez besoin, ça donne des quintes de toux très sèches, et ça vous fait une respiration sifflante qui vous essouffle. Je vois un rapport étroit entre tout ça. (P6, T1)

Caractéristiques des symptômes. Sans sollicitation de la part de l'investigatrice, les participants ont évoqué certaines caractéristiques (p. ex. moment du déclenchement, endroit, qualité) dans la description qu'ils fournissaient de leurs symptômes. La caractéristique la plus couramment employée était la qualité du symptôme (voir le tableau 2). Les descriptions de la douleur, de la fatigue, de l'essoufflement et de l'insomnie étaient assez détaillées, alors que les descriptions des autres symptômes contenaient relativement peu de détails. Par exemple, les participants avaient tendance à décrire à la fois l'emplacement et la qualité de la douleur.

Bon, je me sentais bien avant que la douleur ne commence, dans les côtes, et à l'épaule, et dans le milieu du dos. Ça m'élance, comme s'il y avait du vent qui soufflait sur toute cette partie et quelquefois, ça se promène de mon dos sur mon épaule et de mon épaule sur mon dos. (P8, T1)

Dans la plupart des cas, les participants comparaient la fatigue à une impression générale de ralentissement. Souvent, ils se disaient incapables de faire ce qu'ils pouvaient faire auparavant. 
Par exemple, si j'essaye de faire de l'exercice, je m'essouffle progressivement, mes membres deviennent difficiles à remuer. Vous essayez de continuer à bouger, mais ça va plus lentement, et au bout d'un moment vous ne pouvez plus bouger autant. C'est ce que j'appelle la fatigue. (P9, T1)

Lors de la seconde entrevue, les participants ont parlé d'une sensation générale décrite en terme de certains symptômes qui empiraient. Pour 12 d'entre eux, un symptôme au moins était pire. Leurs descriptions indiquaient que les symptômes s'étaient aggravés et qu'ils leur causaient davantage de difficultés. Les exemples suivants illustrent cette situation :

La fatigue à la première entrevue : Après avoir quitté mon lit pendant une ou deux heures seulement, je sens que mes paupières commencent à tomber. Et en principe, je m'assois juste dans ce fauteuil et je ferme les yeux 5 ou 10 minutes. (P1 T1)

La fatigue à la seconde entrevue : Comme si tout mon corps, de mes doigts jusqu'à mes orteils, vous savez, rien ne veut se bouger. C'est comme, ah! quelquefois quand mes repas arrivent et que je suis couché(e) sur mon lit par exemple, et quelquefois, j'attends 2 ou 3 minutes avant de faire l'effort de m'asseoir et de manger... cette sensation est toujours là. (P1 T2)

La douleur à la première entrevue : Sous le bras et dans la nuque. J'ai la même chose de l'autre côté mais ça ne fait pas mal. Je ne le sens pas comme de ce côté. (P9 T1)

La douleur à la seconde entrevue : Bon, je vais décrire la douleur. La douleur que j'ai quand je suis couché(e), je me repose 3 heures. Après ça, j'ai mal partout. Pas seulement dans la poitrine, mais même sur les côtés, ici aux épaules, et même à la tête, vous savez. (P9 T2)

Impact des symptômes dans la vie de tous les jours. Plus de la moitié des participants $(\mathrm{n}=9)$ lors de la première entrevue et la moitié $(n=8)$ lors de la seconde entrevue ont décrit au moins un symptôme comme interférant avec des activités de la vie quotidienne. La fatigue était le plus souvent ressentie comme un obstacle. Les participants ont indiqué comment les symptômes amenaient à un « ralentissement », altérant la manière dont une activité se faisait auparavant, ou l'empêchant complètement. Pour plusieurs d'entre eux, la présence du symptôme avait un impact sur ce qu'ils pouvaient faire, quand ils pouvaient le faire, et la durée pendant laquelle ils pouvaient maintenir une activité. Par exemple, une femme a expliqué comment elle avait dû changer complètement sa façon de faire les tâches ménagères :

J'ai toujours été quelqu'un qui y va toute la journée. Je travaillais dans une usine. Il fallait que j'y aille et tout d'un coup, je fais un petit peu, la lessive, par exemple, et il faut que je me repose durant la journée, et c'est tout à fait inhabituel pour moi... Je n'y prends plus aucun plaisir. (P11, T1)

Certains participants ont trouvé très déprimant de ne plus arriver à faire les choses qu'ils faisaient autrefois. Un homme a brusquement réalisé à quel point sa capacité de jardiner avait diminué :

J'aime bien jardiner. J'ai toujours aimé jardiner et travailler dehors. J'ai été derrière ma maison à la fin de semaine dernière. Nous avons un rosier le long de la maison, et chaque printemps, il faut que j'arrache les mauvaises herbes et que je nettoie tout, mais j'aime bien faire ça. Jusqu'à maintenant, j'y passais quelques heures un dimanche, mais dimanche dernier, j'ai tenu 20 minutes, c'est tout. Je me suis senti fatigué et complètement à bout de souffle, il a fallu que je m'assoie. (P2 T1)

Les symptômes déclenchant une réponse cognitivelémotionnelle. Les participants ont fourni trois réponses distinctes pour décrire leur vie quotidienne avec les symptômes : 1) la banalisation de la présence d'un symptôme, 2) la frustration et 3) l'inquiétude. La banalisation de la présence des symptômes a été définie par les chercheurs de cette étude comme l'acceptation de leur présence, le désir d'y trouver une explication et la minimisation de leur gravité. Par exemple, un participant a fait part du commentaire suivant : «En général, mon appétit n'est plus ce qu'il était, mais mon activité n'est plus ce qu'elle était»( $\mathrm{P} 4 \mathrm{~T} 1)$. Notons que lors de la première entrevue, les participants n'ont pas jugé que le cancer du poumon était la cause d'un symptôme particulier. Tous les participants $(n=16)$ lors de la première entrevue et 12 lors de la seconde entrevue ont banalisé la présence d'au moins un symptôme (fatigue, perte de poids, perte d'appétit, respiration sifflante, essoufflement, toux).

Le sentiment de frustration était une réponse émotionnelle aux symptômes exprimés dès la première entrevue $(n=6)$. La frustration était prédominante lorsque le symptôme restreignait des activités quotidiennes auxquelles le patient accordait de l'importance. Au cours de la seconde entrevue, une réelle inquiétude se manifestait dans les témoignages de plusieurs participants $(n=6)$. Ces derniers craignaient que les symptômes ne s'aggravent, et ils faisaient le lien avec la progression du cancer du poumon :

J'espère que ça va disparaître, parce que ça se voit sur les radios, et ça m'inquiète un peu, parce que la pleurésie chez quelqu'un qui a un cancer du poumon peut venir des cellules cancéreuses qui s'installent. Je me demande toujours si ça va être pire et naturellement quand j'ai eu ces douleurs qui commencent avec la pleurésie... oh! qu'est-ce qui se passe? Estce qu'il va aussi de l'autre côté? (P1 T2)

\section{Le symptôme le plus incommodant}

La majorité des participants a été capable d'identifier un symptôme comme étant le plus incommodant lors de la première $(n=12)$ et de la seconde $(n=13)$ entrevues (voir le tableau 3$)$. La fatigue était le plus fréquemment citée dans les deux cas. Trois participants ont déclaré ne pas trouver un symptôme plus gênant que les autres. Tous les participants, à l'exception d'un seul, ont décrit, lors de la deuxième entrevue, un symptôme plus gênant différent de celui qu'ils avaient évoqué la première fois. Cependant, la plupart $(n=12)$ ont identifié le symptôme le plus incommodant comme étant le même qu'ils avaient donné en réponse à la question ouverte initiale de l'entrevue, «Ditesmoi comment vous vous êtes senti ces derniers jours. » Cette forme de réponse s'est retrouvée dans les deux entrevues.

Les raisons pour lesquelles les participants considéraient un symptôme plus incommodant que les autres ont été catégorisées dans trois rubriques : impact physique du symptôme (les dimensions
Tableau 2. Caractéristiques des symptômes telles que décrites par les participants

\begin{tabular}{|l|l|l|l|l|l|l|}
\hline \multirow{2}{*}{ Symptôme } & \multicolumn{4}{|l|}{ Nombre de participants rapportant la caractéristique } \\
\cline { 2 - 7 } & Apparition décrite & \multicolumn{2}{l|}{ Siège décrit } & \multicolumn{2}{l|}{ Qualité décrite } \\
\cline { 2 - 7 } & $\begin{array}{l}\text { Première } \\
\text { entrevue }\end{array}$ & $\begin{array}{l}\text { Seconde } \\
\text { entrevue }\end{array}$ & $\begin{array}{l}\text { Première } \\
\text { entrevue }\end{array}$ & $\begin{array}{l}\text { Seconde } \\
\text { entrevue }\end{array}$ & $\begin{array}{l}\text { Première } \\
\text { entrevue }\end{array}$ & $\begin{array}{l}\text { Seconde } \\
\text { entrevue }\end{array}$ \\
\hline Essoufflement & & 5 & & & 7 & 5 \\
\hline Perte d'appétit & & & & & 9 & 8 \\
\hline Fatigue & & & & & 10 & 15 \\
\hline $\begin{array}{l}\text { Respiration } \\
\text { sifflante }\end{array}$ & 6 & & & 10 & 9 & 8 \\
\hline $\begin{array}{l}\text { Douleur } \\
\text { Toux }\end{array}$ & & & 9 & & 6 & 4 \\
\hline Insomnie & & & & & & \\
\hline
\end{tabular}


physiques comprennent l'apparition, le siège, la durée, l'intensité et la qualité du symptôme), l'interférence avec les activités de tous les jours (l'incapacité du participant d'effectuer des activités ordinaires comme les loisirs et les tâches ménagères), et les réponses émotionnelles/cognitives (frustration ou inquiétude; besoin de trouver

\section{Tableau 3.}

Symptôme dégagé comme étant le plus incommodant

\begin{tabular}{|l|l|l|l|}
\hline \multicolumn{2}{|l|}{ Première entrevue } & Seconde entrevue \\
\hline Symptôme & $\begin{array}{l}\text { Nombre de } \\
\text { participants }\end{array}$ & Symptôme & $\begin{array}{l}\text { Nombre de } \\
\text { participants }\end{array}$ \\
\hline Fatigue & 3 & Fatigue & 6 \\
\hline Douleur & 3 & Douleur & 4 \\
\hline Essoufflement & 2 & Perte d'appétit & 1 \\
\hline Insomnie & 2 & Chute de cheveux & 1 \\
\hline Perte d'appétit & 1 & $\begin{array}{l}\text { Faiblesse } \\
\text { des jambes }\end{array}$ & 1 \\
\hline Mucus & 1 & Constipation & 1 \\
\hline & & Vomissements & 1 \\
\hline & & Mal de gorge & 1 \\
\hline
\end{tabular}

Tableau 4.
Stratégies de soins utilisées pour gérer les symptômes

\begin{tabular}{|c|c|c|c|}
\hline \multirow[b]{2}{*}{ Symptôme } & \multirow[b]{2}{*}{ Interventions } & \multicolumn{2}{|c|}{$\begin{array}{l}\text { Nombre de } \\
\text { participants }\end{array}$} \\
\hline & & $\begin{array}{l}\text { Première } \\
\text { entrevue }\end{array}$ & $\begin{array}{l}\text { Seconde } \\
\text { entrevue }\end{array}$ \\
\hline \multirow[t]{2}{*}{ Essoufflement } & Je me repose & 5 & 4 \\
\hline & J'utilise des médicaments & 4 & \\
\hline \multirow[t]{2}{*}{ Perte d'appétit } & Je maintiens une routine & 4 & \\
\hline & Je prends des suppléments & 6 & 5 \\
\hline \multirow[t]{3}{*}{ Fatigue } & Je me repose & 11 & 11 \\
\hline & $\begin{array}{l}\text { Je permets à d'autres } \\
\text { de faire des choses à } \\
\text { ma place }\end{array}$ & & 4 \\
\hline & Je fais de l'exercice & 4 & \\
\hline $\begin{array}{l}\text { Respiration } \\
\text { sifflante }\end{array}$ & Je m'efforce d'expectorer & & 4 \\
\hline \multirow[t]{2}{*}{ Perte de poids } & Je maintiens une routine & 5 & \\
\hline & Je prends des suppléments & 5 & \\
\hline \multirow[t]{2}{*}{ Douleur } & Je change de position & 4 & 4 \\
\hline & J'utilise des médicaments & 9 & 9 \\
\hline Toux & Je m'efforce d'expectorer & 4 & 6 \\
\hline Insomnie & J'utilise des médicaments & 4 & \\
\hline Mucus & Je m'efforce d'expectorer & 8 & 5 \\
\hline
\end{tabular}

une explication à la présence du symptôme). L'interférence avec les activités quotidiennes était la raison généralement évoquée $(n=4)$, qui faisait qu'un symptôme était perçu comme le plus gênant, tandis que l'impact émotionnel était le plus souvent cité $(n=4)$ dans la seconde entrevue. Dans les termes d'un des participants :

Oh! je pense que mes jambes sont le plus grand problème pour le moment. Je suis très inquiet à l'idée de perdre l'usage de mes jambes et quand ça vous arrive, vous êtes pratiquement impotent, vous savez, et vous dépendez tout le temps des autres (P6 T2).

\section{Interventions personnelles des patients}

Lors des deux entrevues, quinze participants ont décrit les stratégies de soins qu'ils utilisaient pour gérer leurs symptômes et celles qu'ils trouvaient les plus efficaces. En général, les participants ont fait état de très peu d'interventions pour gérer les symptômes (voir le résumé au tableau 4). Certaines interventions concernaient plusieurs symptômes. Par exemple, les participants ont mentionnée le repos comme stratégie de soins pour gérer l'essoufflement et la fatigue. La prise de médicaments était utilisée pour gérer plusieurs symptômes, de même que le maintien d'une routine et la délégation de responsabilités à autrui. Par exemple, une participante a parlé de laisser à son époux la responsabilité de faire la cuisine :

Je ne peux pas m'obliger moi-même à faire quoi que ce soit. Normalement, je pouvais me lever et préparer le petit déjeuner le dimanche. Faire des oeufs au bacon, mais c'est lui qui le fait maintenant et je me culpabilise parce qu'il fait beaucoup des choses que je faisais. (P16 T2).

\section{Discussion}

Le but de cette étude pilote était d'amorcer la collecte de points de vue de patients sur les symptômes du cancer du poumon et les stratégies de soins qu'ils utilisaient au moment du diagnostic ainsi que deux mois plus tard. Les résultats de la recherche ont révélé le caractère unique et personnel des témoignages relatifs aux symptômes. On a constaté que les patients utilisaient relativement peu de stratégies face aux multiples symptômes évolutifs qu'ils éprouvaient simultanément. Cette étude pilote avait pour but de déterminer l'efficacité du procédé de recrutement et des méthodes de collecte des données.

En général, les participants n'ont pas fourni de descriptions détaillées de leurs symptômes. Souvent, ils faisaient le lien avec les changements intervenus dans leurs activités de tous les jours et leurs émotions et pensées du moment. Les descriptions de leurs symptômes les amenaient souvent à parler de leur vécu du cancer du poumon. Selon Nerenz et Leventhal (1983), les patients font l'expérience de leur maladie à travers les symptômes, ce qui rend plus difficile la distinction entre les symptômes et l'expérience globale de la maladie. Demander aux patients de parler des symptômes en faisant abstraction de cette expérience, revient à leur demander d'établir une séparation qui pourrait leur paraître artificielle à plusieurs égards

La manière dont les participants à cette étude décrivent leurs symptômes vient étayer la conceptualisation du vécu des symptômes proposée par le Symptom Management Group de l'University of California (1994). Ce groupe considère l'expérience des symptômes comme un processus dynamique impliquant trois dimensions essentielles : la perception du symptôme, l'évaluation du symptôme et la réaction au symptôme. La perception du symptôme fait référence au changement remarqué ou non par une personne dans la manière dont elle se sent habituellement. Lors de la première entrevue, certains ont décrit des changements dans les symptômes qu'ils attribuaient au cancer du poumon, contrairement à d'autres participants. Le second groupe avait souvent éprouvé des symptômes, notamment des symptômes respiratoires, depuis un certain temps avant le diagnostic. Il leur aura été d'autant plus difficile d'isoler des symptômes particuliers comme étant associés au cancer du poumon. Lors de la deuxième entrevue, tous les patients étaient conscients des 
changements intervenus dans les symptômes depuis la première entrevue. L'évaluation des symptômes fait référence aux jugements que les gens portent sur leurs symptômes, tels leur gravité et leurs effets. Les participants mentionnaient souvent que les symptômes ressentis interféraient avec les activités quotidiennes, étaient gênants et, au cours de la seconde entrevue, quels symptômes s'étaient aggravés. La réaction au symptôme comprend à la fois les pensées et les émotions qui s'y rapporte (c.-à-d. la banalisation de la présence du symptôme, le sentiment de frustration et l'inquiétude).

Beaucoup de symptômes décrits dans cette étude ont été rapportés par d'autres chercheurs (Coy et Kennelly, 1980; Brown et al., 1986; Foote, Sexton et Pawlik, 1986; DeMaria et Cohen, 1987; Burt et al., 1990). La fatigue, le symptôme le plus souvent cité, a également été rapportée dans d'autres études (Blesch et al., 1991; Sarna 1993). Bien que l'incidence de la fatigue n'ait pas varié de manière significative entre la première et la deuxième entrevue, ce symptôme a généralement été mentionné comme le plus incommodant lors de la seconde entrevue. Plusieurs raisons peuvent expliquer ce résultat. Tout d'abord, la fatigue a été identifiée comme un symptôme pénible consécutif à la radiothérapie et à la chimiothérapie (Greenberg, Sawicka, Eisenthal et Ross, 1992; Irvine, Vincent, Graydon, Bubela et Thompson, 1994). Dans la présente étude, quatre participants avaient reçu un traitement avant la première entrevue et presque tous les autres, entre la première et la seconde. La fatigue pouvait aussi être la conséquence d'une confrontation quotidienne avec de multiples symptômes. Par exemple, la présence de douleur et d'insomnie pouvait contribuer à la fatigue. Miaskowski, Lee et Dibble (1996) ont mis en évidence une corrélation allant de modérée à forte entre le degré moyen de fatigue quotidienne et l'intensité de la douleur. D'autres chercheurs ont également démontré le lien entre douleur et fatigue (Arathuzik, 1991). Finalement, la progression de la maladie peut également avoir amené les patients à signaler la fatigue comme le symptôme le plus gênant lors de la seconde entrevue. Le cancer du poumon peut être une maladie rapidement débilitante avec le développement et la progression accélérée de symptômes (Boring, Squires, Tong et Montgomery, 1994; Richardson et Johnson, 1993).

Le nombre de fois où les différents symptômes respiratoires étaient signalés comme un tout mérite notre attention. Il était difficile pour les patients de les isoler et de les décrire l'un sans l'autre. Certains sujets ont pu avoir de la difficulté à séparer les symptômes respiratoires, parce que ceux-ci sont perçus comme étroitement liés et en interaction. Il faudra en tenir compte dans l'étude longitudinale.

\section{Stratégies}

Il est quelque peu inquiétant que les participants aient déclaré avoir utilisé aussi peu de stratégies de soins pour gérer leurs symptômes. La prise de médicaments était le plus fréquemment évoquée et, si ces médicaments avaient été efficaces, les patients n'auraient pas éprouvé le besoin d'explorer d'autres interventions (Wilkie et Keefe, 1991). Le repos pour gérer la fatigue était conforme aux observations d'autres équipes de recherche. Graydon, Bubela, Irvine et Vincent (1995) ont relaté les stratégies adoptées de façon autonome par des femmes atteintes du cancer pour gérer la fatigue : s'allonger, s'asseoir ou faire des siestes. À l'heure actuelle, peu de données empiriques sont disponibles à propos des stratégies de gestion de la fatigue chez les sujets atteints de cancer du poumon (Richardson, 1995). Le fait que les participants aient fait état de peu d'interventions pour gérer la dyspnée contredit au moins une étude antérieure, selon laquelle les patients atteints du cancer du poumon recouraient à de nombreuses stratégies (Brown et al. 1986). Cependant, les sujets de l'étude de Brown et collaborateurs avaient des antécédents d'essoufflement avant le diagnostic du cancer du poumon. Cette étude pilote soulève deux questions : les patients ontils eu assez de temps pour apprendre des interventions, étant donné la progression rapide de leur maladie? Avait-on enseigné aux patients des stratégies de gestion de symptômes particulières?
Dans la présente étude, il est impossible de savoir si le faible nombre de stratégies de soins utilisées pour gérer les symptômes reflète la résolution idéale des symptômes ou un répertoire limité d'interventions parmi lesquelles il faut choisir, ou les deux. Des stratégies de gestion des symptômes donnant des résultats peuvent avoir diminué, chez les sujets, le besoin d'explorer d'autres stratégies. Le faible nombre d'interventions employées par les participants pourrait signifier que les symptômes qu'ils éprouvaient n'avaient pas atteint une intensité telle qu'ils nécessitaient des interventions supplémentaires. Peut-être qu'une liste de contrôle des interventions aurait pu être utilisée pour guider les réponses des participants.

\section{Implications pratiques}

En dépit de la faible taille de l'échantillon, il est possible d'envisager plusieurs implications pour la pratique. Pour bien évaluer l'expérience des symptômes chez cette population de patients, les infirmières doivent savoir que les patients sont souvent confrontés à de multiples symptômes qui sont susceptibles d'évoluer avec le temps. Une évaluation continue et permanente des symptômes est importante. Il serait utile d'inclure dans le processus d'évaluation les symptômes tels que la fatigue, la douleur, l'essoufflement, la perte d'appétit, la perte de poids, l'insomnie, la respiration sifflante, la toux et le mucus, souvent mentionnés lors de cette étude.

Les infirmières doivent être attentives à la manière dont les patients parlent de leurs symptômes et les abordent. Elles doivent réaliser que les patients peuvent être incapables de faire des distinctions nettes entre les différents symptômes qu'ils éprouvent. Considérer l'éventail des symptômes décrit par les patients peut aider le personnel soignant à cerner avec précision les symptômes éprouvés et leur impact sur le patient. Une évaluation infirmière, prenant en compte les combinaisons de plusieurs symptômes, peut jouer un rôle important parce qu'une intervention visant à soulager un symptôme particulier pourrait avoir un impact positif ou négatif sur d'autres symptômes.

Lors de l'évaluation des symptômes éprouvés par les patients atteints du cancer du poumon, les infirmières doivent être encouragées à écouter attentivement le récit que fait le patient de son vécu des symptômes. Dans leurs récits, les patients parleront des dimensions physiques de chaque symptôme, de son impact sur les activités quotidiennes et de leurs réponses émotionnelles et cognitives le concernant. Par une écoute attentive, les infirmières se feront une idée précise du quotidien des malades et détermineront la signification des symptômes pour le patient. La compréhension de cette perspective unique leur permettra de fournir une meilleure aide aux patients.

Les stratégies de soins décrites par ces patients pourraient être introduites dans les méthodes d'enseignement au patient. Les stratégies de soins pourraient être partagées avec d'autres patients atteints du cancer du poumon, à la rubrique « Certains sujets atteints d'un cancer du poumon ont trouvé ces stratégies bénéfiques. » Toutefois, l'efficacité de ces stratégies devra être évaluée dans des travaux de recherche futurs.

\section{Implications pour les recherches futures}

Cette étude pilote apporte une information utile sur les défis du recrutement des patients. Les études futures devront se pencher sur les critères d'éligibilité, en particulier celui concernant le lieu de résidence. Nous essayions de réduire la durée des déplacements pour l'investigatrice étant donné que nous voulions réaliser les entrevues en personne. À l'avenir, nous devrons élargir le critère de distance ou permettre les entrevues téléphoniques. 


\section{Références}

Arathuzik, D. (1991). Pain experience for metastatic breast cancer patients. Cancer Nursing, 14, 41-48.

Bailey, A.J., Parma, M.K.B., \& Stephens, R.J. (1998). Physical and psychologic symptoms: Results of the continuous hyperfractionated accelerated radiotherapy (CHART) randomized trial in non-small cell lung cancer. Journal of Clinical Oncology, 16, 3082-3093.

Blesch, K., Paice, J.A., Wickham, R., Harte, N., Schnoor, D.K., Purl, S., et al. (1991). Correlates of fatigue in people with breast or lung cancer. Oncology Nursing Forum, 18, 81-87.

Boring, C.C., Squires, T.S., Tong, T., \& Montgomery, S. (1994). Cancer statistics 1994. CA Cancer Journal for Clinicians, 44(1), 7-26.

Brown, M.L., Carrieri, V., Janson-Bjerklie, S., \& Dodd, M. (1986). Lung cancer and dyspnea: The patient's perspective. Oncology Nursing Forum, 13, 19-24.

Burt, P.A., O’Driscoll, B.R., Notley, H.M., Barber, P.V., \& Stout, R. (1990). Intraluminal irradiation for the palliation of lung cancer with high dose rate micro-selectron. Thorax, 45, 765-768.

Coy, P., \& Kennelly, G.M. (1980). The role of curative radiotherapy in the treatment of lung cancer. Cancer, 45, 698-702.

Degner, L.F., \& Sloan, J.A. (1995). Symptom distress in newly diagnosed ambulatory cancer patients and as a predictor of survival in lung cancer. Journal Pain and Symptom Management, 10(6), 423-31.

DeMaria, L.C., \& Cohen, H.J. (1987). Characteristics of lung cancer in elderly patients. Journal of Gerontology, 42, 540-545.

Edmonds, P., Karlsen, S., Khan, S., \& Addington-Hall, J. (2001). A comparison of the palliative care needs of patients dying from chronic respiratory diseases and lung cancer. Palliative Medicine, 15, 287-295.

Foote, M., Sexton, D.L., \& Pawlik, L. (1986). Dyspnea: A distressing sensation in lung cancer. Oncology Nursing Forum, 13, 25-31.

Graydon, J.E., Bubela, N., Irvine, D., \& Vincent, L. (1995). Fatiguereducing strategies used by patients receiving treatment for cancer. Cancer Nursing, 18, 23-28.

Greenberg, D.B., Sawicka, J., Eisenthal, S., \& Ross, D. (1992). Fatigue syndrome due to localized radiation. Journal of Pain and Symptom Management, 7, 38-45.

Grossman, S.A., Sheidler, V.R., Swedeen, K., Mucenski, J., \& Piantadosi, F. (1991). Correlation of patients and caregiver ratings of cancer pain. Journal of Pain and Symptom Management, 6, 53-57.

Hollen, P.J., Gralla, R.J., Kris, M.G., Eberly, S.W., \& Cox, C. (1999). Normative data and trends in quality of life from the Lung Cancer Symptom Scale (LCSS). Supportive Care Cancer, 7, 140-148.

Holmes, S., \& Eburn, E. (1989). Patients' and nurses' perceptions of symptom distress in cancer. Journal of Advanced Nursing, 14(10), 840-6.
Irvine, D.M., Vincent, L., Graydon, J.E., Bubela, N., \& Thompson, L. (1994). The prevalence and correlates of fatigue in patients receiving treatment with chemotherapy and radiotherapy. Cancer Nursing, 17, 367-378.

Lobchuk, M.M., Krisjanson, L.J., Degner, L.F., Blood, P., \& Sloan, J.A. (1997). Perceptions of symptom distress in lung cancer patients. I: Congruence between patients and family caregivers. Journal of Pain and Symptom Management, 14, 136-146.

Lutz, S.T., Huang, D.T., Ferguson, C.L., Kavanagh, B.D., Tercilla, O.F., \& Lu, J. (1997). A retrospective quality of life analysis using the lung cancer symptom scale in patients treated with palliative radiotherapy for advanced non-small cell lung cancer. International Journal of Oncology, Biology and Physics, 37, 117-122.

Marino, C., Zoppi, M., Morelli, F., Buoncristiano, U., \& Pagni, E. (1986). Pain in early cancer of the lungs. Pain, 27, 57-62.

McCorkle, R., \& Quint-Benoliel, J. (1983). Symptom distress, current concerns and mood disturbances after diagnosis of life-threatening disease. Social Science Medicine, 17, 431-438.

Miaskowski, C., Lee, K.A., \& Dibble, S. (1996). The relationship between pain and fatigue in oncology outpatients. Oncology Nursing Forum, 23(2), 351.

National Cancer Institute of Canada. (2005). Canadian cancer statistics 2005. Toronto, Canada: Author.

Nerenz, D.R., \& Leventhal, H. (1983). Self-regulation theory in chronic illness. In T.G. Burnish \& L.S. Bradley (Eds.), Coping with chronic disease: research and application (pp. 13-37). New York: Academic Press.

Polit, D. F., \& Hungler, B.P. (2003). Nursing research: Principles and methods. Philadelphia: J.B. Lippincott.

Richardson, A. (1995). Fatigue in cancer patients: A review of the literature. European Journal of Cancer Care, 4, 20-32.

Richardson, G. E., \& Johnson, B.E. (1993). The biology of cancer. Seminars in Oncology Nursing, 2, 105-127.

Sarna, L. (1993). Correlates of symptom distress in women with lung cancer. Cancer Practice, 1, 21-28.

Tishelman, C., Degner, L.F., \& Mueller, B. (2000). Measuring symptom distress in patients with lung cancer. Cancer Nursing, 23, 82-90.

University of California, San Francisco, School of Nursing Management, Faculty Symptom Group. (1994). A model for symptom management. Image, 26, 272-276.

Weber, R.P. (1990). Basic content analysis. California: Sage Publications.

Wilkie, D.J., \& Keefe, F.J. (1991). Coping strategies of participants with lung cancer-related pain. Clinical Journal of Pain and Symptom Management, 7, 292-299. 